\title{
Avaliação de Desempenho da Técnica de Transmissão OFDM em Canais de Comunicação via Rede de Distribuição Elétrica de Baixa Tensão
}

\author{
Gilberto de S. Santos ${ }^{1}$, Daniel C. Cunha ${ }^{1}$ e Márcio J. C. Lima ${ }^{2}$
}

\begin{abstract}
Resumo-O presente trabalho tem como objetivo apresentar uma avaliação de desempenho da técnica OFDM aplicada à transmissão de dados via rede externa de distribuição elétrica de baixa tensão, considerando dois tipos de ruídos aditivos: o ruído AWGN e o ruído impulsivo assíncrono. Comparações são feitas alterando-se parâmetros da transmissão (número de subportadoras), do canal PLC (faixa de frequências) e do ruído impulsivo (probabilidade de ocorrência e nível de amplitude).
\end{abstract}

Palavras-Chave - técnica OFDM, rede de distribuição elétrica, ruído impulsivo.

Abstract-The objective of this work is to present a performance evaluation of OFDM technique applied to data transmission through external low voltage powerline network considering two kinds of additive noise: AWGN and assynchronous impulsive one. Comparisons are done by changing of transmission parameters (number of subcarriers), PLC channel (frequency range) and impulsive noise (incidence probability and amplitude level).

Keywords-OFDM technique, powerline network, impulsive noise.

\section{INTRODUÇÃO}

A tecnologia que utiliza a rede de distribuição elétrica como canal de comunicação, conhecida como PLC (do inglês, Powerline Communications), tem por objetivo aproveitar a infraestrutura pré-existente da rede de distribuição elétrica para trafegar sinais de comunicação simultaneamente com os sinais de energia. A coexistência dos dois tipos de sinais se justifica pelo fato de os sinais de comunicações utilizarem uma faixa diferente de frequências $(0,5 \mathrm{MHz}$ a $30 \mathrm{MHz}$ ) [1]. Todavia, o meio físico utilizado, por não ter sido concebido originalmente para comunicações, apresenta-se bastante hostil, no que se refere à presença de interferências e ruídos. Para lidar com essa questão, uma das técnicas empregadas é a multiplexação OFDM (do inglês, Orthogonal Frequency Division Multiplexing), que tem apresentado resultados significativos em canais PLC e em outras aplicações, como por exemplo, os canais ADSL (do inglês, Asymmetric Digital Subscriber Line) [2].

Neste trabalho, é realizada uma avaliação do desempenho da técnica de transmissão OFDM em um canal de comunicação que representa a rede externa de distribuição elétrica de baixa tensão. Na seção II, o modelo matemático do canal é

1 Núcleo de Pesquisa em Telecomunicações, POLI, Universidade de Pernambuco (UPE), Recife-PE, Brasil.

2 Grupo de Pesquisa em Comunicações, Departamento de Eletrônica e Sistemas, Universidade Federal de Pernambuco (UFPE), Recife-PE, Brasil.

E-mails: gilberto.eletronica@gmail.com, dccunha@upe.poli.br e marcio.j.c.lima@gmail.com. Este trabalho foi financiado pelo PIBIC/POLI. devidamente exposto. Na Seção III, resultados de simulação são apresentados. Por fim, na seção IV, são apresentadas as conclusões e as perspectivas de trabalhos futuros.

\section{MODELO DO CANAL}

A Fig. 1(a) ilustra a topologia da rede externa de distribuição elétrica de baixa tensão considerada neste trabalho e cujo modelo matemático foi definido em [3]. O transmissor e o receptor estão localizados nos pontos $\mathrm{A}$ e $\mathrm{C}$, respectivamente. $\mathrm{O}$ ponto $\mathrm{B}$ representa um concentrador PLC e o ponto D é uma derivação da rede, mantida em aberto. Várias reflexões do sinal são causadas ao longo do caminho devido ao descasamento de impedâncias de entrada em cada ponto. Com isso, a propagação do sinal se dá não apenas no caminho direto entre transmissor e receptor, mas também por $(N-1)$ caminhos adicionais. Adicionalmente, é assumido que a atenuação é dependente da frequência, da estrutura da rede e se superpõe ao desvanecimento do canal. Face ao exposto, a função de transferência do canal pode ser descrita pela expressão

$$
H(f)=\sum_{n=1}^{N} g_{n} \cdot e^{-\left(a_{0}+a_{1} f^{k}\right) d_{n}} \cdot e^{-j 2 \pi f \tau_{n}} .
$$

O modelo de canal considerado representa a superposição de sinais a partir de $N$ caminhos distintos, cada um caracterizado individualmente por um fator de ponderação $g_{n}$ e comprimento $d_{n}$. A atenuação é modelada pelos parâmetros $a_{0}, a_{1} \mathrm{e} k$, determinados pelas características físicas dos cabos elétricos e $\tau_{n}$ representa o atraso do $n$-ésimo sinal. Para este trabalho, foram assumidos apenas quatro caminhos de propagação dos sinais $(N=4)$ com os parâmetros da equação (1) definidos em [3]. Portanto, o resultado é um modelo de propagação multipercurso com desvanecimento seletivo em frequência, cuja magnitude da função de transferência $H(f)$, para a faixa de frequências de 0,5 a $20 \mathrm{MHz}$, está ilustrada na Fig. 1(b).

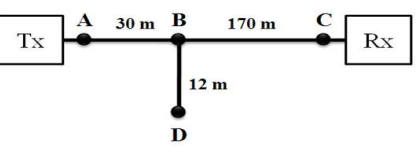

(a)

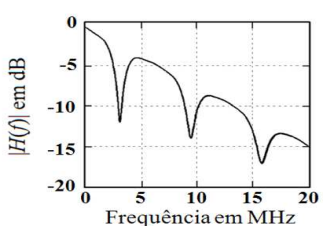

Fig. 1. (a) Topologia da rede. (b) Magnitude de $H(f)$ para o modelo do canal de comunicações adotado.

Os tipos de ruído considerados para o canal adotado foram o ruído AWGN (do inglês, Additive White Gaussian Noise) e o ruído impulsivo assíncrono, sendo este último ocasionado 
por transientes na rede elétrica e uma das principais causas de erros em comunicações através de redes de distribuição elétrica. Desta maneira, o $j$-ésimo sinal recebido na saída do canal pode ser descrito como

$$
r_{j}=s_{j}+w_{j}+i_{j}
$$

em que $s_{j}$ representa um símbolo QPSK (do inglês, Quadrature Phase-Shift Keying) para cada subportadora da técnica OFDM, $w_{j}$ é o ruído AWGN de média zero e variância $\sigma_{w}^{2}$ e $i_{j}$, o ruído impulsivo. A modelagem matemática do ruído impulsivo $i_{j}$ utilizada foi definida em [4], tal que $i_{j}=B_{j} g_{j}$, em que $B_{j}$ é o processo de Bernoulli de probabilidade $p$ e $g_{j}$ é o ruído AWGN de média zero e variância $\sigma_{g}^{2}=\gamma \sigma_{w}^{2}$. $\mathrm{O}$ parâmetro $\gamma$ representa a intensidade do ruído impulsivo, enquanto $p$ representa a sua probabilidade de ocorrência.

\section{RESULTADOS DE SIMULAÇÃO}

Foram realizadas simulações computacionais considerando a transmissão de sinais com a técnica OFDM e o modelo de canal apresentado na Seção II. Na primeira simulação, buscou-se avaliar o desempenho da técnica OFDM no que se refere à variação do número de subportadoras utilizadas. Para tanto, foi adotado apenas o ruído AWGN $w_{j}$. Além disso, toda a faixa de frequência de 0,5 a $20 \mathrm{MHz}$ foi utilizada na transmissão, o que resultou na divisão do espectro total pelo número de subportadoras. A Fig. 2 ilustra o desempenho do sistema por meio da curva de Probabilidade de Erro de Bit $\left(P_{b}\right)$ versus Relação Sinal-Ruído (RSR) em dB.

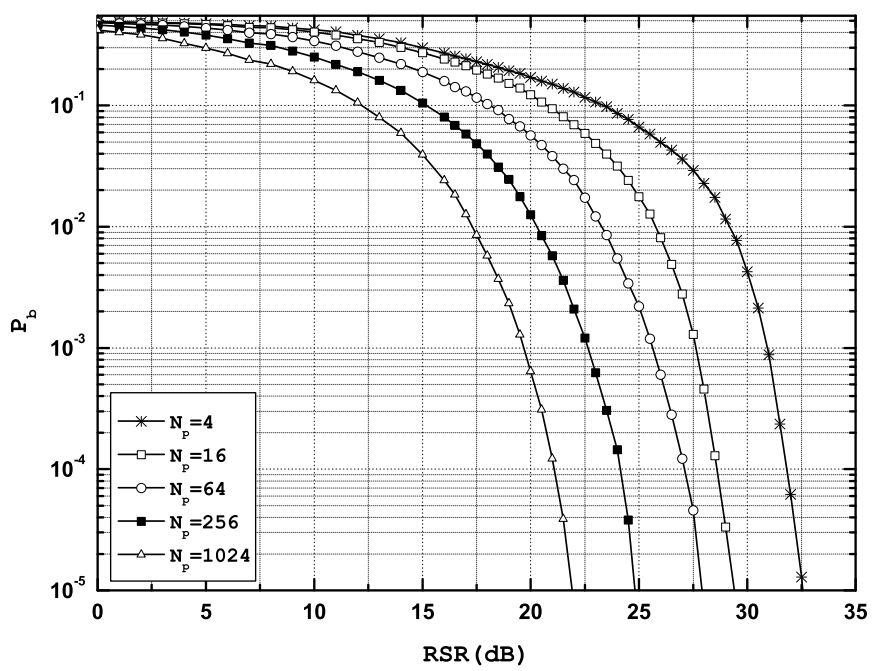

Fig. 2. Probabilidade de Erro de Bit versus Relação Sinal-Ruído da técnica OFDM aplicada ao canal PLC considerando o ruído AWGN e a variação do número de subportadoras $\left(N_{p}\right)$.

Foi observado que a quantidade de bits por subportadora foi diminuída quando aumentou-se o número de subportadoras $\left(N_{p}\right)$, portanto, as subportadoras que sofreram maiores interferências contribuíram com menor ponderação para a $P_{b}$, melhorando o desempenho do sistema. Logo, foram adotadas $N_{p}=1024$ portadoras para a próxima simulação.

$\mathrm{Na}$ segunda simulação, foi considerado não apenas o ruído AWGN $w_{j}$, mas também o ruído impulsivo $i_{j}$. Neste cenário, buscou-se analisar o desempenho da técnica OFDM para diferentes parâmetros $\left(\begin{array}{lll}p & \text { e } & \gamma\end{array}\right)$ do ruído impulsivo.
Adicionalmente, foram consideradas diferentes faixas de frequências para a transmissão OFDM, quais sejam, toda a faixa de frequências de 0,5 a $20 \mathrm{MHz}(\mathrm{TF})$ e as frequências sujeitas às menores atenuações $(0,5$ a $2 \mathrm{MHz})$, denominadas de melhores frequências (MF), como pode ser visto na Fig. 1(b). A Fig. 3 ilustra o desempenho do sistema para as considerações anteriormente mencionadas.

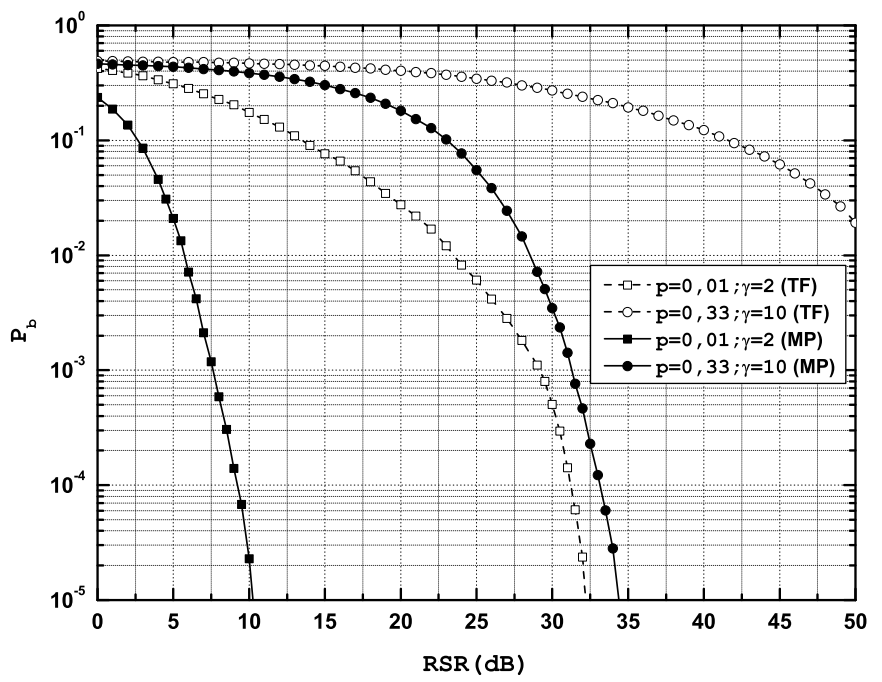

Fig. 3. Probabilidade de Erro de Bit versus Relação Sinal-Ruído da técnica OFDM aplicada ao canal PLC considerando ruído AWGN e ruído impulsivo assíncrono, além da variação da faixa de frequências das subportadoras.

É possível notar que a escolha da faixa de frequências resultou em economia de potência para os dois cenários de ruído impulsivo. No primeiro caso $(p=0,01$ e $\gamma=2)$, para uma $P_{b}=2 \times 10^{-2}$, o ganho de RSR foi de $16,3 \mathrm{~dB}$, enquanto que no segundo ( $p=0,33$ e $\gamma=10$ ), o ganho obtido foi de aproximadamente $22,7 \mathrm{~dB}$.

\section{CONCLUSÕES}

Este trabalho apresentou uma avaliação da técnica de transmissão OFDM em um canal de comunicação que representa a rede externa de distribuição elétrica de baixa tensão. $\mathrm{O}$ desempenho do sistema foi analisado quanto à variação do número de subportadoras e à escolha da faixa de frequências utilizada. $O$ estudo mostrou que $o$ desempenho do sistema evoluiu com o aumento da quantidade de subportadoras, assim como com a escolha da faixa de frequências do canal com menor nível de atenuação. Como perspectiva de trabalhos futuros, sugere-se a investigação deste sistema considerando a inserção de esquemas de codificação de canal, como por exemplo, os códigos turbo e os códigos LDPC (do inglês, Low-Density Parity-Check Codes).

\section{REFERÊNCIAS}

[1] K. Dostert, M. Gotz e R.M. Matthias, "Powerline Channel Characteristics and Their Effect on Communications System Design," IEEE Communications Magazine, pp. 78-86, Abr 2004.

[2] A.R.S. Bahai e B.R. Saltzberg, Multi-Carrier Digital Communications: Theory and Applications of OFDM, 1. ed. New York, 1999. 216p.

[3] M. Zimmermann e K. Dostert, "A Multipath Model for the Powerline Channel," IEEE Transactions on Communications, v. 50, n. 4, pp. 533-559, Abr 2002.

[4] M. Ghosh, "Analysis of the Effect of Impulsive Noise on Multicarrier and Single Carrier QAM Systems," IEEE Transactions on Communications, v. 44, n. 2, pp. 145-147, Fev 1996. 